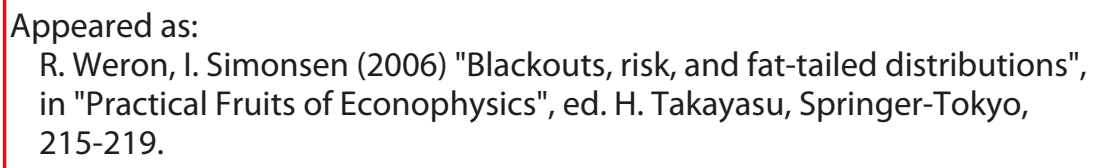

\title{
Blackouts, risk, and fat-tailed distributions
}

\author{
Rafał Weron ${ }^{1}$ and Ingve Simonsen ${ }^{2}$ \\ 1 Hugo Steinhaus Center for Stochastic Methods, \\ Wrocław University of Technology, 50-370 Wrocław, Poland \\ 2 Department of Physics, NTNU, NO-7491 Trondheim, Norway
}

\begin{abstract}
Summary. We analyze a 19-year time series of North American electric power transmission system blackouts. Contrary to previously reported results we find a fatter than exponential decay in the distribution of inter-occurrence times and evidence of seasonal dependence in the number of events. Our findings question the use of self-organized criticality, and in particular the sandpile model, as a paradigm of blackout dynamics in power transmission systems. Hopefully, though, they will provide guidelines to more accurate models for evaluation of blackout risk.
\end{abstract}

Electric power transmission networks are complex systems. ${ }^{1}$ Due to economic factors, they are commonly run near their operational limits. Major cascading disturbances or blackouts of these transmission systems have serious consequences. Although, each blackout can be attributed to a particular cause: natural peril, equipment malfunction or human behavior, an exclusive focus on the causes of these events can overlook the global dynamics of a complex system. Instead, it might be interesting to study blackouts from a top-down perspective. Following Carreras et al. (2004) we analyze a time series of blackouts to explore the nature of these complex systems. However, despite the fact that we are using the same database we obtain different results. Consequently, we challenge their arguments that lead to modeling blackouts as a self-organized criticality (SOC) phenomenon (Bak et al., 1987).

The reliability events - like the August 1996 blackout in Northwestern America that disconnected 30,390 MW of power to 7.5 million customers or the even more spectacular August 2003 blackout in Northeastern America that disconnected $61,800 \mathrm{MW}$ of power to 50 million people - demonstrate that the necessary operating practices, regulatory policies, and technological tools for dealing with the changes are not yet in place to assure an acceptable level of reliability. In a restructured environment, prices are a matter of private choice, yet the reliability of the delivery system affects everyone.

\footnotetext{
${ }^{1}$ For a brief review of approaches to complex systems and cascading failure in power system blackouts see Dobson et al. (2004).
} 
Naturally, the operation of the electric system is more difficult to coordinate in a competitive environment, where a much larger number of parties are participating. For example, in North America about one-half of all domestic generation is now sold over ever-increasing distances on the wholesale market before it is delivered to customers (Albert et al., 2004). Consequently the power grid is witnessing power flows in unprecedented magnitudes and directions. Unfortunately, it seems that the development of reliability management reforms and operating procedures has lagged behind economic reforms in the power industry. In addition, responsibility for reliability management has been disaggregated to multiple institutions (Carrier et al., 2000). All this results in an increase of the risk of blackouts, not only in North America, but also world-wide.

The Disturbance Analysis Working Group (DAWG) database ${ }^{2}$ summarizes disturbances that have occurred in the electric systems in North America. The database is based on major electric utility system disturbances reported to the U.S. Department of Energy (DOE) and the North American Electrical Reliability Council (NERC). The data arise from government incident reporting requirements criteria detailed in DOE form EIA-417.

Carreras et al. (2004) analyzed the first 15 years of data (1984-1998) from the DAWG database. As currently four more years of data are available ${ }^{3}$ we study two datasets: D98 covering the period 1984-1998 and D02 covering the full data set 1984-2002. The first one is used for comparison with the previous findings, while the second lets us extend the analysis and draw more upto-date conclusions. The data are of diverse magnitude and of varying causes (including natural perils, human error, equipment malfunction, and sabotage). It is not clear how complete these data are, but it seems to be the bestdocumented source for blackouts in the North American power transmission system. Besides the date and the region of occurrence, two measures of the event's severity are given: the amount of power lost (in MW) and the number of customers affected.

There are 435 documented blackouts in the first 15 years (dataset D98), which gives on average 29 blackouts per year. A few events have missing data in one or both of the severity fields. For the analysis of blackout sizes we have used only those 427 occurrences which have complete data in both columns. ${ }^{4}$ The average inter-occurrence time is 12.6 days, but the blackouts are distributed over the 15 years in a non-uniform manner with a maximum waiting time of 252 days between event origins. Furthermore, the mean and the maximum restoration times are 14 hours and 14 days, respectively, indicating

\footnotetext{
${ }^{2}$ Publicly available from http://www.nerc.com/ dawg/database.html.

3 The delay in data distribution is due to the complexity of the problem. It can take months after a large blackout to dig through the records, establish the events occurring and reproduce a causal sequence of events.

${ }^{4}$ However, for the waiting time distribution analysis we have used all occurrences. A preprocessed, spreadsheet-ready ASCII format datafile is available from http://www.im.pwr.wroc.pl/ rweron/exchlink.html.
} 

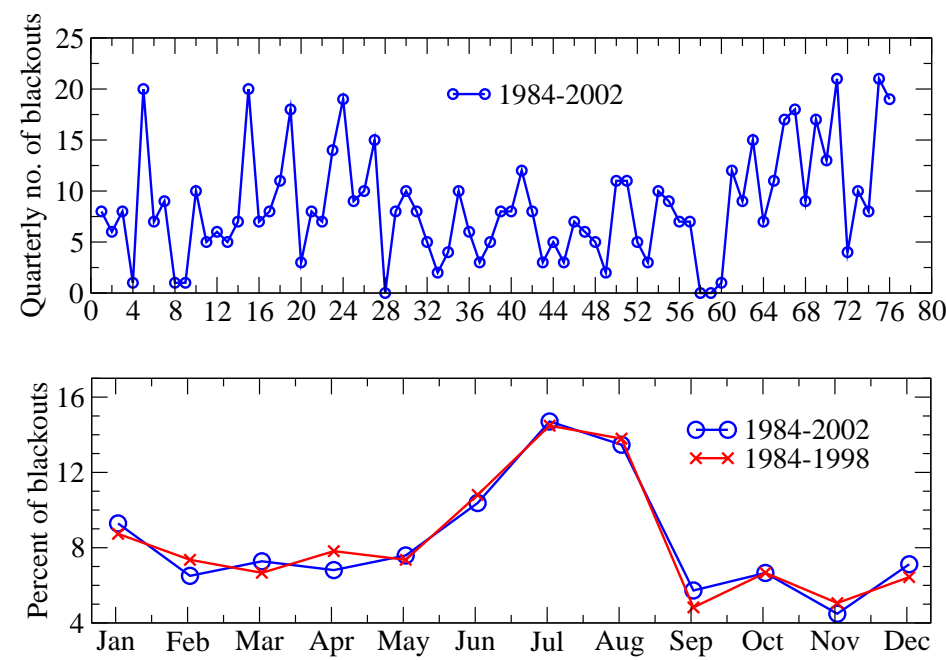

Fig. 1. The quarterly number of blackout events from 1984 till 2002 (top) and annual distribution of monthly events (bottom) for the North-American power grid.

that the inter-occurrence times are more or less equivalent to the quiet times (the lapses of time between the end of a blackout and the beginning of the next one).

In the full dataset (D02) there are 646 documented blackouts, yielding on average 34 blackouts per year. However, only 578 occurrences have complete severity data, since - especially in 1999 and 2000 - there are many missing values. The average period of time between blackouts is now only 10.7 days, indicating a recent increasing trend in the number of blackouts, while the mean and the maximum restoration times are slightly higher: 16 hours and 15 days, respectively.

Although the scarcity of data limits sound statistical inference, looking at the top panel of Fig. 1 we can intuitively divide the dataset into three parts: an initial period of relatively volatile activity (1984-1990; quarters 1-28), followed by a fairly calm period (1991-1998; quarters 29-60), and, most recently, a period of increasing activity (1999-2002; quarters 61-76). Whether this is a consequence of deregulation, different incident reporting procedures or simply randomness remains an open question. However, the seasonal behavior of the outages is indisputable. Roughly $30 \%$ of all blackouts take place in July and August, see the bottom panel of Fig. 1, regardless of the dataset analyzed. Our observations contradict earlier reports, where the authors detected no evidence of systematic changes in the number of blackouts or (quasi-)periodic behavior (Carreras et al., 2004).

A closer inspection of the waiting times between blackouts reveals a nontrivial nature. The distribution does not have an exponential tail, as reported 


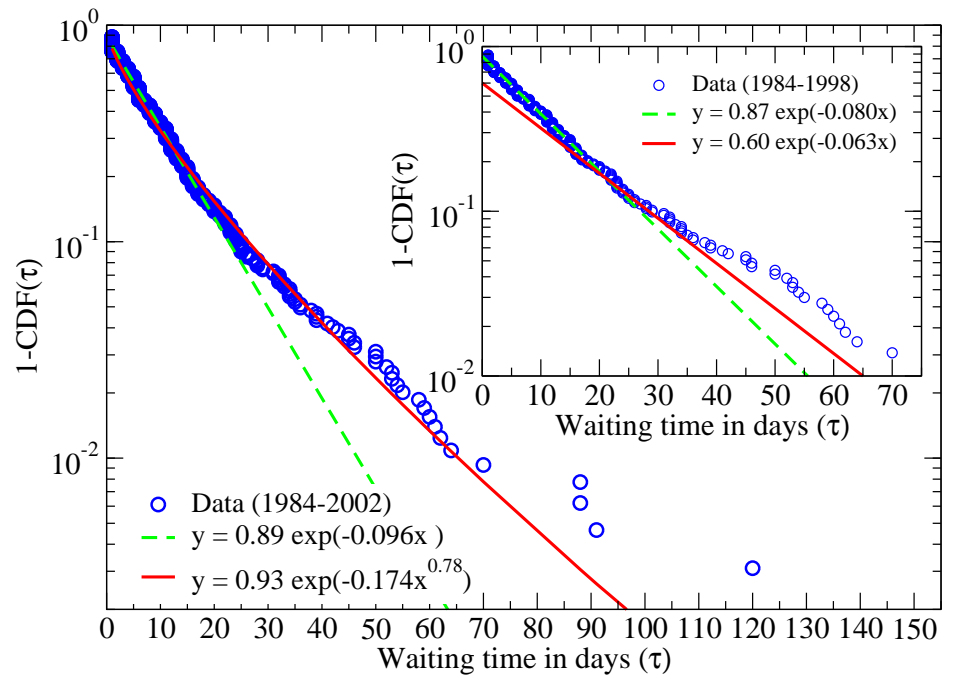

Fig. 2. The complementary cumulative distribution function $(1-\operatorname{CDF}(\tau))$ of the waiting times $\tau$ (measured in days) between two consecutive blackout origins for the North-American power transmission system using the D02 (main panel) and D98 data sets (inset). The dashed lines represent exponential fits to the distributions. The solid lines correspond to a stretch exponential fit (main panel) and the exponential fit obtained by Carreras et al. (2004) using the same data set (inset).

e.g. by Chen et al. (2001), but rather a fatter one. ${ }^{5}$ As can be seen in Fig. 2 the deviation is significant for both D98 and D02. These findings question the SOC-type approach to modeling blackout dynamics (Carreras et al., 2004) since SOC-type dynamics should exhibit exponential decay in the waiting time distribution (Boffetta et al., 1999, Carreras et al., 2004).

It is apparent that large blackouts, as the mentioned earlier August 1996 and August 2003 events, are rarer than small blackouts. But how much rarer are they? Analysis of the D98 and D02 datasets shows that the complementary cumulative probability distribution of the blackout sizes does not decrease exponentially with the size of the outage, but rather has a power-law tail of exponent $\alpha=1$, see Fig.3. Hence, if we evaluate the risk of a blackout as the product of its frequency and cost (commonly regarded to be proportional to unserved energy, see e.g. Billinton and Allan (1996)), then the total risk associated with the large blackouts is - due to the power-law type distribution of blackout sizes - much greater than the risk of small outages. This is strong motivation for investigating the global dynamics of series of blackouts that can lead to power-law tails. The investigated models, though, should take into account all or at least most of the characteristics revealed in this study.

\footnotetext{
${ }^{5}$ Waiting time distribution of high-frequency financial data show similar fatterthan exponential distributions (Scalas et al., 2005).
} 


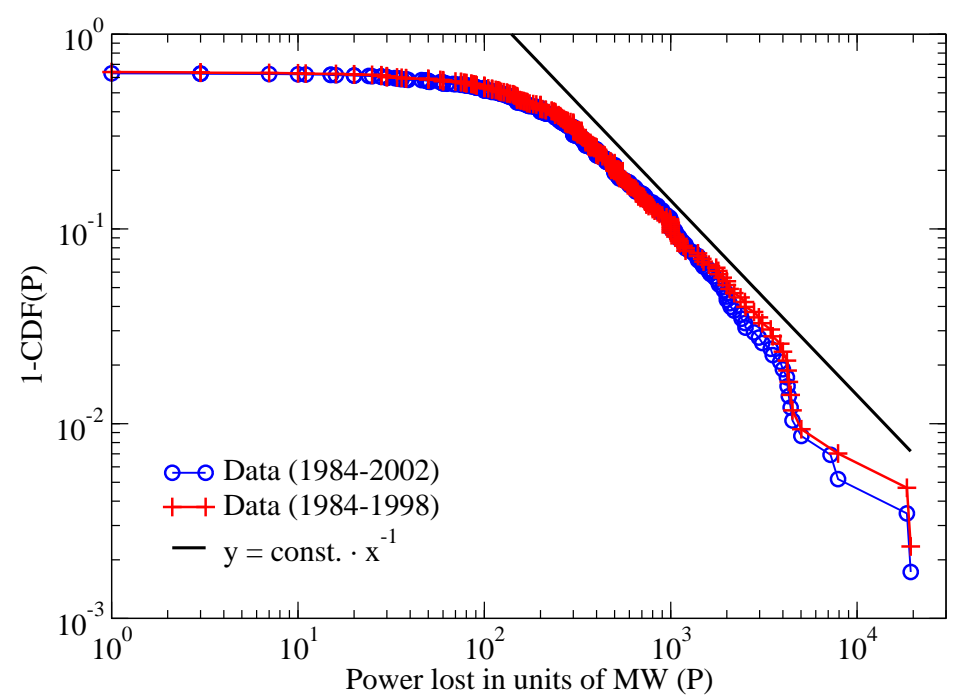

Fig. 3. The complimentary cumulative distribution $(1-\mathrm{CDF}(\mathrm{P}))$ of power lost $(\mathrm{P})$ due to blackouts for the North-American electric power transmission system.

\section{References}

Albert, R., Albert, I., Nakarado, G.L. (2004) Structural vulnerability of the North American power grid. Phys. Rev. E 69, 025103(R).

Bak, P., Tang, C., Wiesenfeld, K. (1987) Self-organized criticality: an explanation of $1 /$ f noise. Phys. Rev. Lett. 59, 381-384.

Billinton, R., Allan, R.N. (1996) Reliability evaluation of power systems, 2nd ed. Plenum Press, New York.

Boffetta, G., Carbone, V., Guliani, P., Veltri, P., Vulpiani, A. (1999) Power laws in solar flares: Self-organized criticality or turbulence? Phys. Rev. Lett. 83, 4662-4665.

Carreras, B.A., Newman, D.E., Dobson, I., Poole, A.B. (2004) Evidence for self-organized criticality in a time series of electric power system blackouts. IEEE Trans. Circuits and Systems 51(9), 1733-1740.

Carrier, P., et al. (2000) Findings from the summer of 1999. Interim Report of the U.S. Department of Energy's Power Outage Study Team.

Chen, J., Thorp, J.S., Parashar, M. (2001) Analysis of electric power system disturbance data. In: Proc. 34th Hawaii Int. Conf. on System Sciences, Maui.

Dobson, I., Carreras, B.A., Lynch, V., Newman, D.E. (2004) Complex systems analysis of series of blackouts: Cascading failure, criticality, and self-organization. Proc. Bulk Power System Dynamics \& Control - VI, Cortina d'Ampezzo.

Scalas, E., Gorenflo, R., Luckock, H., Mainardi, F., Mantelli, M., Raberto, M. (2005) Anomalous waiting times in high-frequency financial data. Quantitative Finance, in print. See also: arXiv:cond-mat/0310305. 